\title{
Modified Taylor series method for solving nonlinear differential equations with mixed boundary conditions defined on finite intervals
}

\author{
Hector Vazquez-Leal ${ }^{*}$, Brahim Benhammouda², Uriel Antonio Filobello-Nino ${ }^{1}$, Arturo Sarmiento-Reyes ${ }^{3}$, \\ Victor Manuel Jimenez-Fernandez ${ }^{1}$, Antonio Marin-Hernandez ${ }^{4}$, Agustin Leobardo Herrera-May ${ }^{5}$, Alejandro \\ Diaz-Sanchez ${ }^{3}$ and Jesus Huerta-Chua ${ }^{6}$
}

\begin{abstract}
In this article, we propose the application of a modified Taylor series method (MTSM) for the approximation of nonlinear problems described on finite intervals. The issue of Taylor series method with mixed boundary conditions is circumvented using shooting constants and extra derivatives of the problem. In order to show the benefits of this proposal, three different kinds of problems are solved: three-point boundary valued problem (BVP) of third-order with a hyperbolic sine nonlinearity, two-point BVP for a second-order nonlinear differential equation with an exponential nonlinearity, and a two-point BVP for a third-order nonlinear differential equation with a radical nonlinearity. The result shows that the MTSM method is capable to generate easily computable and highly accurate approximations for nonlinear equations.
\end{abstract}

AMS Subject Classification: $34 L 30$

Keywords: Taylor series method; Boundary valued problems; Shooting technique; Dirichlet conditions; Mixed boundary conditions

\section{Introduction}

Nonlinear differential equations are a popular tool to model complicated dynamical phenomenons of many branches of sciences. Unfortunately, a drawback of this approach arises when exact solutions are required but not available. On one side, pure numerical methods employed to solve nonlinear differential equations can exhibit numerical instabilities, oscillations or false equilibrium states, among others. On the other side, approximative methods are a good option when semi-analytic solutions are required. Some examples of such methods are: homotopy perturbation method (HPM) (FilobelloNino et al. 2012a; Filobello-Nino et al. 2012b; He 1999; 2009; Khan et al. 2013; Vazquez-Leal 2012; Vazquez-Leal et al. 2012a; Vazquez-Leal et al. 2012b; Vazquez-Leal et al. 2012c), homotopy analysis method (HAM) (Hassana

\footnotetext{
*Correspondence: hvazquez@uv.mx

1 Electronic Instrumentation and Atmospheric Sciences School, Universidad Veracruzana, Cto. Gonzalo Aguirre Beltrán S/N, 91000 Xalapa, Mexico Full list of author information is available at the end of the article
}

and El-Tawil 2011; He 2004; Tan and Abbasbandy 2008), variational iteration method (VIM) (Chang 2010; Khan et al. 2012), Taylor series method (TSM) (Barrio et al. 2011; Rodriguez and Barrio 2012; Shiraishi et al. 2011; Wazwaz 1998), Adomian decomposition method (Duan and Rach 2011; Wazwaz 1998), among others. Nevertheless, TSM highlights because of its simplicity and power; it does not require a perturbation parameter as the perturbation based techniques or trial functions as HAM or HPM does. In addition, TSM is straightforward and can be programmed using computer algebra packages like Maple or Mathematica. What is more, TSM method was conceived as a tool to solve differential equations governed by Dirichlet conditions (DC), although, mixed boundary condition $(\mathrm{MBC})$ problems are important and common in several fields of physics. Therefore, in this work, we propose a modified Taylor series method (MTSM) for MBC problems that is based on the induction of shooting constants (SC) (Stoer and Bulirsch 2002). Such constants arise from two sources: 
1. The conversion of $\mathrm{MBC}$ to $\mathrm{DC}$. This process implies the choosing of an expansion point (usually at zero) and the conversion of the $\mathrm{MBC}$ that are not at the expansion point to $\mathrm{SC}$ constants to be determined later by MTSM. The number of these constants is the same as the number of boundary conditions that are not at the expansion point.

2. Increasing the order of the original differential equation by the application of extra derivatives; as a strategy to add integration constants to solution that work as shooting constants or adjustment parameters. The number of this SC constants depends of the level of accuracy that we require from the approximate MTSM solution. For all the cases study of this work only one extra derivative is required to obtain a good fitting with respect to the exact solution, although it depends of the particular problem under study.

The aforementioned combined shooting technique of MTSM aids to circumvent the issue of TSM method with MBC. In order to show the benefits of this proposal, three nonlinear problems described with $\mathrm{MBC}$ on finite intervals are solved: three-point BVP for a thirdorder nonlinear differential equation with a hyperbolic sine nonlinearity (Duan and Rach 2011), two-point BVP for a second-order nonlinear differential equation with an exponential nonlinearity (Duan and Rach 2011; Scott and Vandevender 1975) and a two-point BVP for the third-order nonlinear differential equation with a radical nonlinearity (Duan and Rach 2011).

This paper is organized as follows. In Section 'MTSM method', we introduce the basic idea of MTSM method. In Section 'Cases study', we show the solution procedure for three nonlinear problems. Numerical simulations and a discussion about the results are provided in Section 'Numerical simulation and discussion'. Finally, a brief conclusion is given in Section 'Conclusion'.

\section{MTSM method}

We consider a nonlinear differential equation expressed as

$$
u^{(n)}=N(u)-f(x), \quad x \in \Omega,
$$

having as boundary condition

$$
B\left(u, \frac{\partial u}{\partial \eta}\right)=0, \quad x \in \Gamma,
$$

where $n$ is the order of the differential equation, $N$ is a general operator; $f(x)$ is a known analytic function, $B$ is a boundary operator, $\Gamma$ is the boundary of domain $\Omega$, and $\partial u / \partial \eta$ denotes differentiation along the normal drawn outwards from $\Omega$.
Firstly, we increase the order of the differential equation

$$
u^{(n+k)}=\frac{d^{k}}{d x^{k}}[N(u)-f(x)], \quad x \in \Omega,
$$

where $k$ is a constant related to the number of the desired $\mathrm{SC}$ constants.

Next, it is possible to express the Taylor series solution for (3) as

$$
\begin{aligned}
u_{T}= & u\left(x_{0}\right)+\frac{u^{\prime}\left(x_{0}\right)}{1 !}\left(x-x_{0}\right)^{1}+\frac{u^{\prime \prime}\left(x_{0}\right)}{2 !}\left(x-x_{0}\right)^{2} \\
& +\frac{u^{\prime \prime \prime}\left(x_{0}\right)}{3 !}\left(x-x_{0}\right)^{3}+\frac{u^{(4)}\left(x_{0}\right)}{4 !}\left(x-x_{0}\right)^{4}+\cdots,
\end{aligned}
$$

where $x_{0}$ is the expansion point and derivatives $u^{(i)}\left(x_{0}\right)$ $(i=0,1, \ldots)$ are expressed in terms of the parameters and boundary conditions of (3).

As we require to solve $\mathrm{MBC}$ problems, the boundary conditions not located at the chosen expansion point $x_{0}$ will be replaced by shooting constants giving as result traditional DC conditions. Next, in order to obtain the coefficients of $(4)\left(u^{(i)}\left(x_{0}\right), i=0,1, \ldots\right)$, MTSM requires (I) calculate the successive derivatives of (3) and (II) evaluate each derivative using the Dirichlet conditions. Finally, in order to fulfil the boundary conditions originally replaced by the SC constants is necessary to evaluate (4) in such points; then, the resulting system of equations is solved to obtain the value of the SC constants. It is important to remark that the order of the Taylor expansion (4) is chosen in order to include all the shooting constants in the polynomial; as long as we satisfy such condition the order of the Taylor expansion can be increased to improve accuracy.

The constants due to the extra $k$-derivatives (see (3)) are applied to minimize the mean square residual (MSR) error defined as

$$
\int_{x_{i}}^{x_{f}}\left(u_{T}^{(n)}-N\left(u_{T}\right)+f(x)\right)^{2} d x,
$$

where $u_{T}$ is the approximated TSM solution (4), and $\left[x_{i}, x_{f}\right]$ is the finite interval delimited by the MBC.

\section{Cases study}

In the present section, we will solve three cases study to show the utility of the MTSM method to solve nonlinear problems. For all cases study the expansion point of TSM is at $x_{0}=0$ and the derivatives were performed using Maple 17 software. 


\section{Third-order nonlinear equation}

Consider the three-point BVP for the third-order nonlinear differential equation with a hyperbolic sine nonlinearity (Duan and Rach 2011)

$$
\begin{aligned}
& u^{\prime \prime \prime}-1-x \sinh (u)=0, \quad u(0)=0, u(0.25)=1, u(1)=0, \\
& 0 \leq x \leq 1
\end{aligned}
$$

where prime denotes derivative with respect to $x$ and the exact solution is unknown.

Firstly, we derive (6), resulting

$$
\begin{aligned}
& u^{(i v)}-x u^{\prime} \cosh (u)-\sinh (u)=0, \\
& u(0)=0, u^{\prime}(0)=c_{1}, u^{\prime \prime}(0)=c_{2}, u^{\prime \prime \prime}(0)=c_{3}, 0 \leq x \leq 1,
\end{aligned}
$$

where the boundary conditions of (6) are replaced by its Dirichlet conditions accordingly to the increased order.

Next, we resolve (7) for $u^{(i v)}$ and perform successive derivatives, resulting

$$
\begin{aligned}
& u^{(i v)}=x u^{\prime} \cosh (u)+\sinh (u), \\
& u^{(v)}=2 u^{\prime} \cosh (u)+x\left(u^{\prime}\right)^{2} \sinh (u)+x u^{\prime \prime} \cosh (u), \\
& u^{(v i)}=3\left(u^{\prime}\right)^{2} \sinh (u)+3 u^{\prime \prime} \cosh (u)+\cdots
\end{aligned}
$$

Now, the boundary conditions of (7) are substituted into (8), yielding

$$
\begin{aligned}
& u^{(i v)}(0)=0, \\
& u^{(v)}(0)=2 c_{1}, \\
& u^{(v i)}(0)=3 c_{2},
\end{aligned}
$$

Finally, using (9) and the initial conditions of (7), we can formulate the sixth-order Taylor series expansion (see (4))

$$
u_{T}(x)=c_{1} x+\frac{1}{2} c_{2} x^{2}+\frac{1}{6} c_{3} x^{3}+\frac{1}{60} c_{1} x^{5}+\frac{1}{240} c_{2} x^{6} .
$$

Finally, if we substitute the boundary conditions $u(0.25)=1$ and $u(1)=0$ into (10) and solve for the shooting constants $\left(c_{1}\right.$ and $\left.c_{2}\right)$, it results

$$
\begin{aligned}
& c_{1}=5.347659477+0.04132001785 c_{3}, \\
& c_{2}=-10.78371002-0.4139015236 c_{3} .
\end{aligned}
$$

Next, $c_{3}$ is used as adjustment parameter to minimize the mean square residual (MSR) error by resolving

$$
\frac{\partial}{\partial c_{3}}\left[\int_{0}^{1}\left(u_{T}^{\prime \prime \prime}-1-x \sinh \left(u_{T}\right)\right)^{2} d x\right]=0,
$$

where $c_{1}$ and $c_{2}$ were previously substituted by (11). It is important to notice that in order to obtain a symbolic expression for $c_{3}$ the hyperbolic sine was replaced by its fifth-order Taylor series.

The result of solving (12) is $c_{3}=1.1353380202$ giving a minimum MSR error of

$$
\int_{0}^{1}\left(u_{T}^{\prime \prime \prime}-1-x \sinh \left(u_{T}\right)\right)^{2} d x=0.007337036421
$$

\section{Second-order nonlinear differential equation}

Consider the two-point BVP second-order nonlinear differential equation with an exponential nonlinearity (Duan and Rach 2011; Scott and Vandevender 1975)

$$
u^{\prime \prime}-\exp (u)=0, \quad u(0)=0, u(1)=0, \quad 0 \leq x \leq 1,
$$

where prime denotes derivative with respect to $x$ and exact solution is

$$
\begin{aligned}
u(x) & =2 \ln \left(C \sec \left(\frac{C(2 x-1)}{4}\right)\right)-\ln (2), \\
C & =1.336055694906108
\end{aligned}
$$

Now, we derive (14), resulting

$$
\begin{aligned}
& u^{\prime \prime \prime}-u^{\prime} \exp (u)=0, \quad u(0)=0, u^{\prime}(0)=c_{1}, u^{\prime \prime}(0)=c_{2} \\
& 0 \leq x \leq 1
\end{aligned}
$$

where the boundary conditions of (14) are replaced by its Dirichlet conditions accordingly to the increased differential equation order.

As the aforementioned procedure for first case study, we obtain the following sixth-order Taylor series

$$
\begin{aligned}
u_{T}(x)= & c_{1} x+\frac{1}{2} c_{2} x^{2}+\frac{1}{6} c_{1} x^{3}+\frac{1}{24}\left(c_{1}^{2}+c_{2}\right) x^{4} \\
& +\frac{1}{120}\left(c_{1}^{3}+3 c_{1} c_{2}+c_{1}\right) x^{5} \\
& +\frac{1}{720}\left(c_{1}^{4}+6 c_{1}^{2} c_{2}+5 c_{1}^{2}+3 c_{2}^{2}+c_{2}\right) x^{6} .
\end{aligned}
$$


Finally, if we substitute the boundary condition $u(1)=0$ into (17) and solve for the shooting constant $c_{2}$, it results

$$
\begin{aligned}
c_{2}= & -c_{1}^{2}-3 c_{1}-\frac{391}{6} \\
& +\frac{1}{6} \sqrt{24 c_{1}^{4}+144 c_{1}^{3}+4596 c_{1}^{2}+3924 c_{1}+152881},
\end{aligned}
$$

where the negative square root term was discarded because it did not minimize the mean square residual error.

Repeating the procedure for first case study (see (12)), we find that $c_{1}=-0.4582864419$ minimize the MSR error, resulting

$$
\int_{0}^{1}\left(u_{T}^{\prime \prime}-\exp \left(u_{T}\right)\right)^{2} d x=0.0004389212651,
$$

where $u_{T}$ corresponds to (17).

\section{Third-order nonlinear differential equation with a radical nonlinearity}

Consider the two-point BVP for the third-order nonlinear differential equation with a radical nonlinearity (Duan and Rach 2011)

$$
\begin{aligned}
& u^{\prime \prime \prime}+\sqrt{1-u^{2}}=0, \quad u(0)=0, u^{\prime}(0)=1, u(\pi / 2)=1 \\
& 0 \leq x \leq \pi / 2
\end{aligned}
$$

where prime denotes derivative with respect to $x$ and exact solution is

$$
u(x)=\sin (x)
$$

Firstly, we derive (20), resulting

$$
\begin{aligned}
& u^{(i v)}-\frac{u u^{\prime}}{\sqrt{1-u^{2}}}=0, \\
& u(0)=0, u^{\prime}(0)=1, u^{\prime \prime}(0)=c_{1}, u^{\prime \prime \prime}(0)=c_{2}, \quad 0 \leq x \leq \pi / 2,
\end{aligned}
$$

where the boundary conditions of (20) are replaced by its Dirichlet conditions accordingly to the increased order.

As the aforementioned procedure for first case study, we obtain the following sixth-order Taylor series

$$
u_{T}(x)=x+\frac{1}{2} c_{1} x^{2}+\frac{1}{6} c_{2} x^{3}+\frac{1}{120} x^{5}+\frac{1}{240} c_{1} x^{6} .
$$

Finally, if we substitute the boundary condition $u(\pi / 2)=1$ into (23) and solve for the shooting constant $c_{2}$, it results

$$
c_{2}=-\frac{1}{320} \frac{c_{1} \pi^{6}+4 \pi^{5}+1920 c_{1} \pi^{2}+7680 \pi-15360}{\pi^{3}} .
$$

Repeating the procedure for first case study (see (12)), we find that $c_{1}=0.001065300514$ minimizes the MSR error, resulting

$$
\int_{0}^{\pi / 2}\left(u_{T}^{\prime \prime \prime}+\sqrt{1-u_{T}^{2}}\right)^{2} d x=0.0001811801833
$$

where $u_{T}$ corresponds to (23).

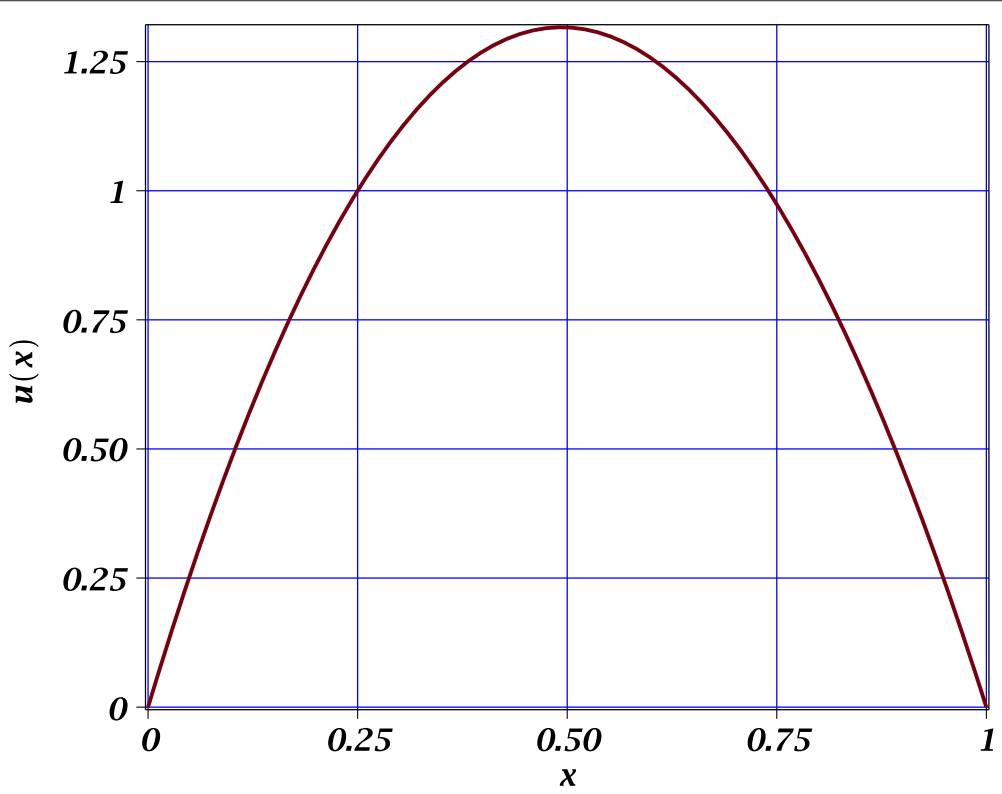

Figure 1 MTSM approximation (10) of (6). The MSR error is 0.007337036421. 


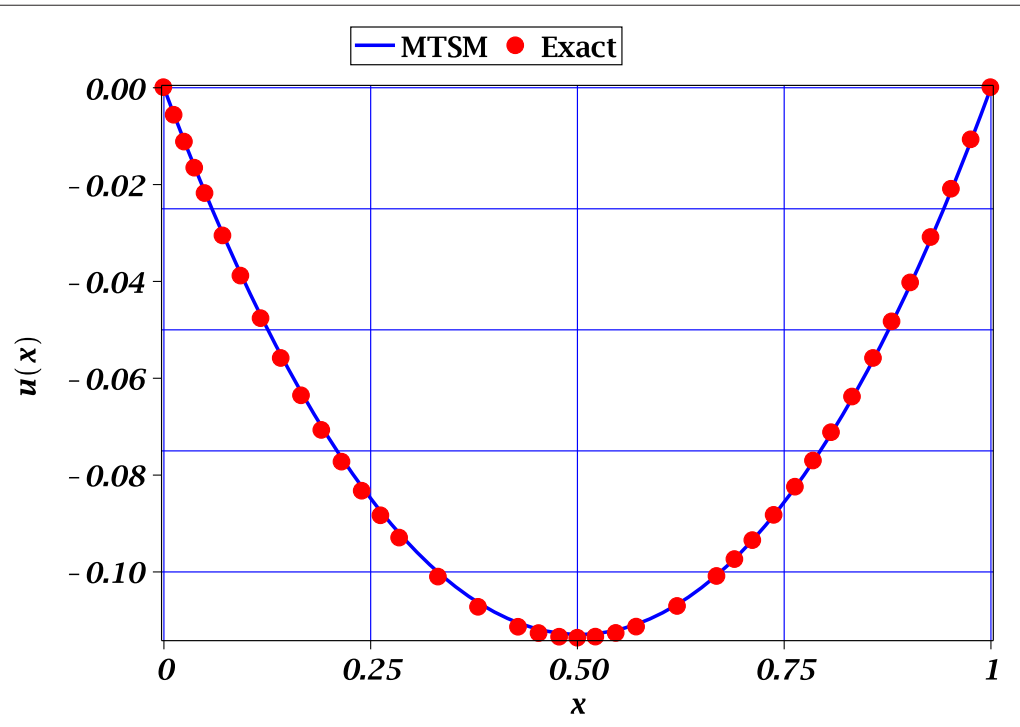

Figure 2 Exact solution (15) (solid circles) and approximate MTSM solution (17) (solid line) for (14). The MSR error is 0.0004389212651.

\section{Numerical simulation and discussion}

From Figures 1, 2 and 3, we observe the high accuracy of the proposed MTSM approximations for all cases study. For all cases study, only one extra derivative was required to obtain an acceptable low MSR error. It is important to mention that for Figures 2 and 3 the comparison is of MTSM approximations are versus the exact solutions (see (15) and (21)) depicting the accuracy of the approximations. Nonetheless, the problem (6) do not possess a known exact solution and the build-in professional numerical routines for BVP problems from Maple does failed to deal with this problem. Therefore, the only reference to know the error from exact solution is the MSR error of 0.007337036421 (see (13)), which is considered very low. This means that (10) is highly accurate.

The usefulness of coupling of a shooting method (Stoer and Bulirsch 2002) along with extra derivatives and the TSM method was exhibited by the solution of different highly nonlinear boundary value problems expressed in terms of nonlinearities such as: high order derivatives combined with hyperbolic sine, exponential and radical terms, among others. What is more, the shooting

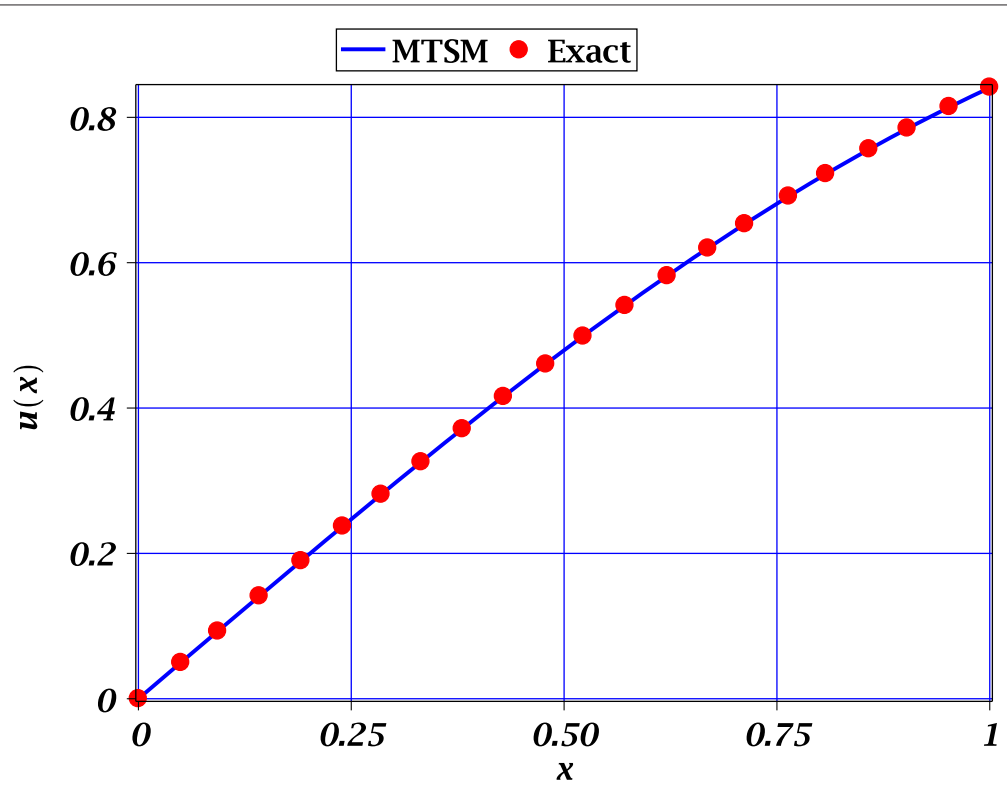

Figure 3 Exact solution (21) (solid circles) and approximate MTSM solution (23) (solid line) of (20). The MSR error is 0.0001811801833. 
constants were used to fulfil the boundary conditions originally discarded by the artificial Dirichlet conditions. Finally, an extra derivative induced an extra shooting constant to minimize the MSR error giving as result high accurate (see (13), (19) and (25)) handy power series solutions. Finally, if users require more accurate approximated solutions, they should augment the number of $\mathrm{SC}$ constants (increasing $k$ ) to improve the potential of minimizing the MSR error (5).

In this work, we presented a modified Taylor series method to deal with nonlinear problems exhibiting mixed boundary conditions defined on finite intervals. The aforementioned procedure and results show that MTSM can obtain power series solutions using only derivatives without requiring to solve a system of differential equations or the proposal of trial functions as HPM ( He 1999; 2009) or HAM (He 2004; Tan and Abbasbandy 2008) methods, or an iterative solution procedure of integrals as VIM (Chang 2010) method. In addition, MTSM is not based on the existence of a perturbation parameter (Filobello-Nino et al. 2013). Therefore, further work will address more potential applications of the proposed method to other type of problems or inclusive other type of boundary conditions as: Robin or Neumann.

\section{Conclusion}

This work introduced the application of a modified Taylor series method (MTSM) for solving boundary value problems (BVPs) with mixed boundary conditions defined on a finite interval. We were able to obtain accurate, easy computable, handy approximations for all cases study. The shooting constants arising from the substitution of the mixed boundary conditions by Dirichlet conditions and the extra derivatives of the differential equation demonstrate - with examples - to be a powerful strategy that provides easy computable and accurate approximations. In addition, more extra derivatives can be applied to the differential equation to increase the number of shooting/adjustment constants, giving as result an enhanced convergence of the MTSM method.

\section{Competing interests}

The authors declare that they have no competing interests.

\section{Authors' contributions}

All authors contributed extensively in the development and completion of this article. All authors read and approved the final manuscript.

\section{Acknowledgements}

We gratefully acknowledge the financial support from the National Council for Science and Technology of Mexico (CONACyT) through grant CB-2010-01 \#157024. The author would like to thank Roberto Castaneda-Sheissa, Rogelio-Alejandro Callejas-Molina, and Roberto Ruiz-Gomez for their contribution to this project.

\section{Author details}

${ }^{1}$ Electronic Instrumentation and Atmospheric Sciences School, Universidad Veracruzana, Cto. Gonzalo Aguirre Beltrán S/N, 91000 Xalapa, Mexico. ${ }^{2}$ Higher Colleges of Technology, Abu Dhabi Men's College, P.O. Box 25035 Abu Dhabi, United Arab Emirates. ${ }^{3}$ National Institute for Astrophysics, Optics and Electronics, Luis Enrique Erro No. 1, Sta. Maria, 72840 Tonantzintla, Puebla, Mexico. ${ }^{4}$ Department of Artificial Intelligence, Universidad Veracruzana, Sebastián Camacho 5 Centro, 91000 Xalapa, Veracruz, Mexico. ${ }^{5}$ Micro and Nanotechnology Research Center, Universidad Veracruzana, Calzada Ruiz Cortines 455, Boca del Rio, 94292 Veracruz, Mexico. ${ }^{6}$ Facultad de Ingenieria Civil, Universidad Veracruzana, Venustiano Carranza S/N, Col. Revolucion, 93390 Poza Rica, Veracruz, Mexico.

Received: 19 December 2013 Accepted: 19 March 2014

Published: 25 March 2014

\section{References}

Barrio R, Rodriguez M, Abad A, Blesa F (2011) Breaking the limits: the taylor series method. Appl Math Comput 217(20): 7940-7954

Chang SH (2010) A variational iteration method for solving troesch's problem. J Comput Appl Math 234(10): 3043-3047

Duan J-S, Rach R (2011) A new modification of the adomian decomposition method for solving boundary value problems for higher order nonlinear differential equations. Appl Math Comput 218(8): 4090-4118

Filobello-Nino U, Vazquez-Leal H, Khan Y, Castaneda-Sheissa R, Yildirim A, Hernandez-Martinez L, Sanchez-Orea J, Castaneda-Sheissa R, Bernal FR (2012a) Hpm applied to solve nonlinear circuits: a study case. Appl Math Sci 6(85-88): 4331-4344

Filobello-Nino U, Vazquez-Leal H, Castaneda-Sheissa R, Yildirim A, Hernandez-Martinez L, Pereyra-Diaz D, Perez-Sesma A, Hoyos-Reyes C (2012b) An approximate solution of blasius equation by using hpm method. Asian J Math Stat 5: 50-59

Filobello-Nino U, Vazquez-Leal H, Khan Y, Yildirim A, Jimenez-Fernandez VM, Herrera-May AL, Castaneda-Sheissa R, Cervantes-Perez J (2013) Perturbation method and laplace-padé approximation to solve nonlinear problems. Miskolc Math Notes 14(1): 89-101

Hassana HN, El-Tawil MA (2011) An efficient analytic approach for solving two-point nonlinear boundary value problems by homotopy analysis method. Math Methods Appl Sci 34: 977-989

He J-H (1999) Homotopy perturbation technique. Comput Methods Appl Mech Eng 178(34): 257-262

He J-H (2004) Comparison of homotopy perturbation method and homotopy analysis method. Appl Math Comput 156(2): 527-539

$\mathrm{He} \mathrm{J}-\mathrm{H}$ (2009) An elementary introduction to the homotopy perturbation method. Comput Math Appl 57(3): 410-412

Khan Y, Vazquez-Leal H, Hernandez-Martinez L, Faraz N (2012) Variational iteration algorithm-ii for solving linear and non-linear odes. Int J Phys Sci 7(25): 3099-4002

Khan Y, Vazquez-Leal H, Wu Q (2013) An efficient iterated method for mathematical biology model. Neural Comput Appl 23(3-4): 677-682. doi:10.1007/s00521-012-0952-z

Rodriguez M, Barrio R (2012) Reducing rounding errors and achieving brouwers law with taylor series method. Appl Numerical Math 62(8): 1014-1024

Scott MR, Vandevender WH (1975) A comparison of several invariant imbedding algorithms for the solution of two-point boundary-value problems. Appl Math Comput 1(3): 187-218

Stoer J, Bulirsch R (2002) Introduction to numerical analysis. 3rd edn. Springer, New York

Shiraishi F, Egashira M, Iwata M (2011) Highly accurate computation of dynamic sensitivities in metabolic reaction systems by a taylor series method. Math Biosci 233(1): 59-67

Tan Y, Abbasbandy S (2008) Homotopy analysis method for quadratic riccati differential equation. Commun Nonlinear Sci Numerical Simul 13(3): 539-546

Vazquez-Leal H (2012) Rational homotopy perturbation method. J Appl Math 2012: 1-14. doi:10.1155/2012/490342

Vazquez-Leal H, Filobello-Nino U, Castaneda-Sheissa R, Hernandez-Martinez L, Sarmiento-Reyes A (2012a) Modified hpms inspired by homotopy continuation methods. Math Probl Eng 2012: 19

Vazquez-Leal H, Sarmiento-Reyes A, Khan Y, Filobello-Nino U, Diaz-Sanchez A (2012b) Rational biparameter homotopy perturbation method and 
laplace-padé coupled version. J Appl Math 2012: 1-21. doi:10.1155/2012/923975

Vazquez-Leal H, Castañeda-Sheissa R, Filobello-Niño U, Sarmiento-Reyes A, Sánchez-Orea J (2012c) High accurate simple approximation of normal distribution related integrals. Math Probl Eng 2012: 22

Wazwaz A-M (1998) A comparison between adomian decomposition method and taylor series method in the series solutions. Appl Math Comput 97(1): 37-44

doi:10.1186/2193-1801-3-160

Cite this article as: Vazquez-Leal et al:: Modified Taylor series method for solving nonlinear differential equations with mixed boundary conditions defined on finite intervals. SpringerPlus 2014 3:160.

Submit your manuscript to a SpringerOpen ${ }^{\circ}$ journal and benefit from:

- Convenient online submission

- Rigorous peer review

- Immediate publication on acceptance

- Open access: articles freely available online

- High visibility within the field

- Retaining the copyright to your article

Submit your next manuscript at $\boldsymbol{~ s p r i n g e r o p e n . c o m ~}$ 